\title{
Dimensionless ratios: Characteristics of quantum liquids and their phase transitions
}

\author{
Yi-Cong Yu, ${ }^{1}$ Yang-Yang Chen, ${ }^{1}$ Hai-Qing Lin, ${ }^{2, *}$ Rudolf A. Römer, ${ }^{3}$ and Xi-Wen Guan ${ }^{1,4,5, \dagger}$ \\ ${ }^{1}$ State Key Laboratory of Magnetic Resonance and Atomic and Molecular Physics, Wuhan Institute of Physics and Mathematics, \\ Chinese Academy of Sciences, Wuhan 430071, China \\ ${ }^{2}$ Beijing Computational Science Research Center, Beijing 100094, China \\ ${ }^{3}$ Department of Physics and Centre for Scientific Computing, University of Warwick, Coventry, CV4 7AL, United Kingdom \\ ${ }^{4}$ Center for Cold Atom Physics, Chinese Academy of Sciences, Wuhan 430071, China \\ ${ }^{5}$ Department of Theoretical Physics, Research School of Physics and Engineering, Australian National University, \\ Canberra ACT 0200, Australia
}

(Received 30 November 2015; revised manuscript received 22 October 2016; published 15 November 2016)

\begin{abstract}
Dimensionless ratios of physical properties can characterize low-temperature phases in a wide variety of materials. As such, the Wilson ratio (WR), the Kadowaki-Woods ratio, and the Wiedemann-Franz law capture essential features of Fermi liquids in metals, heavy fermions, etc. Here we prove that the phases of many-body interacting multicomponent quantum liquids in one dimension (1D) can be described by WRs based on the compressibility, susceptibility, and specific heat associated with each component. These WRs arise due to additivity rules within subsystems reminiscent of the rules for multiresistor networks in series and parallel-a novel and useful characteristic of multicomponent Tomonaga-Luttinger liquids (TLL) independent of microscopic details of the systems. Using experimentally realized multispecies cold atomic gases as examples, we prove that the Wilson ratios uniquely identify phases of TLL, while providing universal scaling relations at the boundaries between phases. Their values within a phase are solely determined by the stiffnesses and sound velocities of subsystems and identify the internal degrees of freedom of said phase such as its spin degeneracy. This finding can be directly applied to a wide range of 1D many-body systems and reveals deep physical insights into recent experimental measurements of the universal thermodynamics in ultracold atoms and spins.
\end{abstract}

DOI: 10.1103/PhysRevB.94.195129

\section{INTRODUCTION}

One of the central challenges in condensed matter physics is to understand how different phases of matter can arise and how these phases can be characterized. Many phenomena such as, e.g., superconductivity, magnetism, and quantum phase transitions in strongly correlated systems [1], BoseEinstein condensation of dilute gases [2] and of excitons in semiconductors, electronic transport in low-dimensional systems, and heavy-fermion physics [3], are known to exist due to the collective nature of the underlying many-body processes. Collective phenomena are particularly strong in low-dimensional systems where the reduced dimensionality enhances the interaction of elementary constituents [4-7]. In order to characterize the various phases, dimensionless ratios such as the celebrated Wiedemann-Franz (WF) law [8] or the Kadowaki-Wood ratio $[9,10]$ are very useful. They usually involve ratios of measurable quantities which stem from similar underlying processes.

The WF law is universal across a wide range of materials and temperature regimes because nonuniversal contributions due to, e.g., density of states and effective mass often cancel out. Conversely, deviations from the WF law can be used to characterize the emergence of new physical processes [11]. Remarkably, recent new experiments [12] show that the WF law holds even at quantum phase transitions. Similarly, the

\footnotetext{
*haiqing0@csrc.ac.cn

†xiwen.guan@anu.edu.au
}

Wilson ratio (WR) [13,14],

$$
R_{\mathrm{W}}^{\chi}=\frac{4}{3}\left(\frac{\pi k_{B}}{\mu_{B} g_{\text {Lande }}}\right)^{2} \frac{\chi}{c_{V} / T},
$$

between the susceptibility $\chi$ and the specific heat $c_{V}$ divided by the temperature $T$, measures the strength in magnetic fluctuations versus thermal fluctuations $[3,15,16]$. Here $k_{B}$ is Boltzmann's constant, $\mu_{B}$ is the Bohr magneton, and $g_{\text {Lande }}$ is the Lande factor. This dimensionless ratio has been observed in a wide variety of Kondo systems [3,14]. Recent studies of the WR for magnetic states of a 1D spin ladder compound [17] and the two-component attractive Fermi gas [18] show that $R_{\mathrm{W}}^{\chi}$ allows a convenient identification of magnetic phases. Dimensionless ratios therefore provide an elegant experimental and theoretical approach to a qualitative and quantitative characterization of the nature of complex multicomponent quantum liquids.

In Fig. 1, we show that the Wilson-like ratio

$$
R_{\mathrm{W}}^{\kappa}=\frac{\pi^{2} k_{B}^{2}}{3} \frac{\kappa}{c_{V} / T},
$$

relating particle fluctuations to energy fluctuations, is even more successful in determining phases in quantum liquids. Recent experiment [19] show that the compressibility WR (2) determines the Luttinger parameter for the phase of TLL in 1D Bose gases and characterizes the quantum fluctuations at quantum criticality. The key observation underlying the predictive strength of both WRs is that the phases are related to simple additivity rules of the underlying elementary excitations. In this paper, we shall prove that such additivity rules are in fact general for multicomponent quantum liquids 


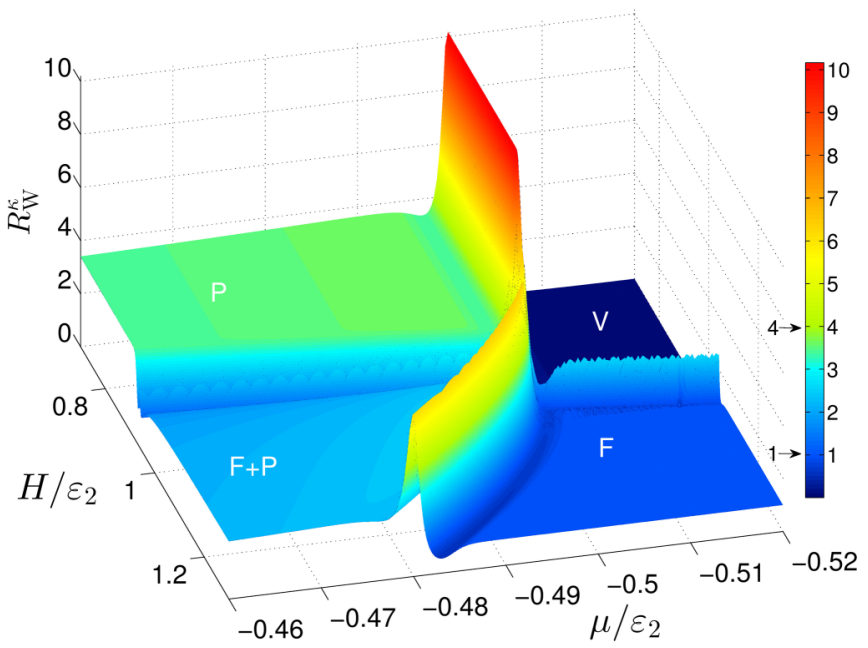

FIG. 1. Phase diagram for the attractive SU(2) Yang-Gaudin model at $T=0.001 \varepsilon_{2} / k_{B}$ given as a 3D plot of the WR $R_{W}^{\kappa}$ in the $\mu-H$ plane. The pair binding energy is denoted by $\varepsilon_{2}=0.5$ and the interaction strength $g_{1 \mathrm{D}}=-2$. The expected values of $R_{W}^{\kappa}=1,4$ in the excess fermion phase $(\mathrm{F})$ and the pure pair phase $(\mathrm{P})$ in the strong coupling limit, respectively, are indicated in the legend on the right. The mixed phase of fermions and pairs is denoted as $\mathrm{F}+\mathrm{P}$.

in 1D-and we expect that they also hold for multicomponent Fermi liquids in higher dimensions [16]. We further show that both WRs stem from these exact additivity rules, can quantitatively identify quantum phases of Tomonaga-Luttinger liquids (TLL) independent of microscopic details, and exhibit universal scaling behavior at the quantum transitions between phases. Their values within a given phase give information about the internal degrees of freedom of a phase, e.g., its spin degeneracy. Such information is important, e.g., for the collective nature of multicomponent interacting quantum liquids in cold atoms [20-23], large-symmetry fermionic systems [24,25], spin chains [17,26], and the 1D Hubbard model [5].

\section{THEORY}

\section{A. General considerations}

In order to show the versatility of the WRs in identifying phases, we are interested in systems that have a rich phase diagram. We therefore start by studying first an attractive quantum liquid that supports a hierarchy of bound states. The system shall consist of unbound particles, pairs of particles, triples of particles and so on until we have at most $w$-tuples. For convenience, we shall denote a bound state formed from $r$ particles as a $r$ complex. Let $N_{r}$ denote the number of $r$ complexes which have formed. Then the total number of particles is given as $N=N_{1}+\ldots+w N_{w}$ and the density of an $r$ complex is $n_{r}=N_{r} / L$ such that the total particle density is $n=\sum_{r=1}^{w} r n_{r}$. The relative propensity of a phase is governed by a set of external fields $H_{r}, r=1, \ldots, w$ with $H_{w}=0$. Here the $H_{r}$ are coupling to the (spin) moment of each $r$ complex.
Then we can write for the Hamiltonian of the system

$$
\mathcal{H}=\mathcal{T}+\mathcal{V}-\sum_{r=1}^{w-1} H_{r} N_{r}-\mu N,
$$

where $\mathcal{T}$ and $\mathcal{V}$ are as of yet unspecified kinetic and many-body interaction energies.

In order to compute the WRs (1) and (2), we need to compute the susceptibility $\chi$, the compressibility $\kappa$, and specific heat $c_{V}$ for the $w$-component system in the Gibbs ensemble $G\left(\mu, H_{1}, \ldots, H_{w}\right)$. In a single component system, $\chi$ and $\kappa$ can be straightforwardly computed as $\partial M / \partial H$ (with magnetization $M$ ) for a constant particle number (canonical) or $\partial n / \partial \mu$ for constant external fields (grand canonical). For a multicomponent system, we therefore define in complete analogy a chemical potential $\mu_{r}$ for each of the $r$ complexes via

$$
\mu_{r}=\mu+\frac{1}{r} H_{r}+\frac{\varepsilon_{r}}{r},
$$

where $\varepsilon_{r}$ denotes the binding energy of an $r$ complex. Remarkably, the quantity $\mu_{2}$ has already been measured in a recent experimental study of the equation of state for $2 \mathrm{D}$ ultracold fermions [23]. It gives a deep physical insight into the crossover from Bose-Einstein condensate to Bardeen-CooperSchrieffer superconductor. Indeed, the choice (4) allows us to define a Fermi energy at $T=0$ for each fluid of $r$ complexes in the same way that $\epsilon_{F}$ is defined in the Landau's Fermi liquid picture. We now introduce a stiffness in grand canonical and canonical ensembles, respectively, as

$$
D_{r}^{\kappa}=\frac{r}{\hbar \pi}\left(\frac{\partial \mu_{r}}{\partial n_{r}}\right)_{H_{1}, \ldots, H_{w-1}}, \quad D_{r, r^{\prime}}^{\chi}=\frac{r}{\hbar \pi}\left(\frac{\partial \mu_{r}}{\partial n_{r^{\prime}}}\right)_{n}
$$

for a $r$-complex fluid subject to $\mu$ and the field $H_{r^{\prime}}$. Also, the sound velocity for $r$ complexes is defined as usual via $v_{r}=$ $\left.\frac{d \epsilon_{r}(k)}{d k}\right|_{k_{F}}$, where $\epsilon_{r}(k)$ is the dispersion relation for $r$ complexes and $k_{F}$ is the Fermi momentum. With these definitions, we can then prove in 1D that the individual $\kappa_{r}, \chi_{r}$, and $c_{V, r}$ in terms of the $D_{r}^{\kappa}, D_{r, r^{\prime}}^{\chi}$, and $v_{r}$ are given as

$$
\kappa_{r}=\frac{r^{2}}{\pi \hbar} \frac{1}{D_{r}^{\kappa}}, \quad \chi_{r, r^{\prime}}=\frac{r^{2}}{\pi \hbar} \frac{1}{D_{r, r^{\prime}}^{\chi}}, \quad c_{V, r}=\frac{\pi k_{B}^{2} T}{3 \hbar} \frac{1}{v_{r}}
$$

as shown in the Appendix. We note that similar relations hold in Fermi liquids (FLs) [16]. Our strategy therefore is to derive the densities $n_{r}$, the chemical potentials $\mu_{r}$, and the dispersions $\epsilon_{r}(k)$ for the $r$ complexes as functions of $H_{1}, \ldots, H_{w}$ and $\mu$. We expect that the definitions (5) and (6) are useful in general for interacting quantum liquids. However, analytical or numerical access to these quantities is not necessarily straightforward. Following a series of recent papers [27], we prove here that integrable 1D multicomponent systems allow the explicit construction of $n_{r}, \mu_{r}$, and $\epsilon_{r}$ using the thermodynamic Bethe ansatz (TBA) [28-30]. Following earlier theoretical [16] and recent experimental [19,23] findings, we furthermore speculate that similar relations also hold for FLs in higher dimensions and expect that such effective chemical potentials for multicomponent systems can therefore serve as convenient handles to describe multicomponent quantum liquids. 


\section{B. The $w$-component quantum liquid}

Let us now consider a specific $w$-component Hamiltonian (3) that supports multicomponent quantum liquids. A convenient and experimentally relevant example is the 1D $\mathrm{SU}(w)$ Fermi gas with $\delta$-function interaction confined to length $L[31,32]$. The system is described by the Hamiltonian (3) with

$$
\mathcal{T}+\mathcal{V}=-\frac{\hbar^{2}}{2 m} \sum_{i=1}^{N} \frac{\partial^{2}}{\partial x_{i}^{2}}+g_{1 \mathrm{D}} \sum_{1 \leqslant i<j \leqslant N} \delta\left(x_{i}-x_{j}\right)
$$

and with the chemical potential $\mu$ and the effective Zeeman energy $E_{z}=\sum_{r=1}^{w} \frac{1}{2} r(w-r) n_{r} H_{r}$. There are $w$ possible hyperfine states $|1\rangle,|2\rangle, \ldots,|w\rangle$ that the fermions can occupy. Experimentally, $g_{1 \mathrm{D}}\left(=-2 \hbar^{2} / m a_{1 \mathrm{D}}\right.$, with $a_{1 \mathrm{D}}$ the effective scattering length in 1D [33]; from now on, we choose our units such that $\hbar^{2}=2 m=1$ unless we particularly use the units) can be tuned from a weak interaction to a strong coupling regime via Feshbach resonances. This model provides an ideal experimental testing ground to probe few- and many-body physics [34-36].

For the system described by (7), the relation (4) follows naturally from the structure of the TBA equations [27] (see Appendix A). We will use in addition general energy-transfer relations for breaking a $w$ complex into smaller $r$ complexes, i.e.,

$$
\frac{1}{r} H_{r}=\mu_{r}-\mu_{w}+\frac{1}{w} \varepsilon_{w}-\frac{1}{r} \varepsilon_{r}
$$

with $r=1,2, \ldots, w-1$. Note that $\varepsilon_{r}=\frac{1}{48} r\left(r^{2}-1\right) g_{1 \mathrm{D}}^{2}$ is the explicit binding energy for each $r$ complex in the system (7). Using the energy and particle conservation conditions, (8) and $n=\sum_{r=1}^{w} r n_{r}$, respectively, we find that $\kappa$ and $\chi_{r}$ obey the additivity rules,

$$
\begin{gathered}
\kappa=\kappa_{1}+\kappa_{2}+\cdots+\kappa_{w}, \\
\frac{1}{\chi_{r}}=\frac{1}{\chi_{r, 1}}+\frac{1}{\chi_{r, 2}}+\cdots+\frac{1}{\chi_{r, w}},
\end{gathered}
$$

in the TLL phases (see Appendix B). Here the susceptibility $\chi_{r}$ represents the responses of different bound states to the change of the field $H_{r}$. Such additivity appears naturally for a noninteracting fluid. Determining $v_{r}$ from the TBA, we similarly find that

$$
c_{V}=c_{V, 1}+c_{V, 2}+\cdots+c_{V, w} .
$$

Based on the rules given in (9), (10), and (11), we can construct the WRs of the $w$-component $\mathrm{SU}(w)$ system to be

$$
\begin{aligned}
R_{\mathrm{W}, r^{\prime}}^{\chi} & =\left(\sum_{r=1}^{w} \frac{D_{r, r^{\prime}}^{\chi}}{r^{2}}\right)^{-1}\left(\sum_{r=1}^{w} \frac{1}{v_{r}}\right)^{-1}, \\
R_{\mathrm{W}}^{\kappa} & =\left(\sum_{r=1}^{w} \frac{r^{2}}{D_{r}^{\kappa}}\right)\left(\sum_{r=1}^{w} \frac{1}{v_{r}}\right)^{-1} .
\end{aligned}
$$

These ratios are dimensionless and uniquely determined by the sound velocities and stiffnesses. We note that the form of the WRs in (12) and (13) is similar for a $w$-component FL; all interaction effects have been included into (8) via the choice of $\mu_{r}$.
In the strong coupling regime, $R_{\mathrm{W}}^{\kappa}$ for a pure $r$-complex phase $\left(R_{\mathrm{W}}^{\chi}=0\right.$ in pure phases [18]) can be given in the form

$$
R_{W}^{\kappa, r}=r K_{r}=r^{2}\left(1-2 B_{r} \frac{1}{|\gamma|}+B_{r}^{2} \frac{1}{\gamma^{2}}\right)+O\left(\gamma^{-3}\right),
$$

where $\gamma=g_{1 \mathrm{D}} / 2 n$ is the dimensionless interaction strength, $B_{1}=0$, and $B_{r}=\sum_{k=1}^{r-1} 1 / k$ with $r=2, \ldots, w$. In the above equation, $K_{r}$ is the phenomenological Luttinger parameter which can be directly measured through the Wilson ratio $R_{\mathrm{W}}^{\chi}$. This provides an important way to test the low energy Luttinger theory. For $\gamma \rightarrow \infty, R_{W}^{\kappa}$ then displays plateaus at the integers $1^{2}, \ldots, r^{2}, \ldots, w^{2}$. Deviations from integer $r^{2}=1,4,9$ occur not because of a weakness of the ratios introduced here but because of the physics underlying and determining the stability and mixture of bound states in a given phase. Thus $R_{W}^{\kappa}$ provides a convenient quantitative phase characteristic for quantum systems at finite $T$ as well as at $T \rightarrow 0$, see Fig. 1 and below.

\section{Scaling of the WRs}

It is particularly interesting that the WRs identify non-TLL behavior and quantum criticality in the quantum critical regime. In fact, the WRs show sudden enhancement near a quantum phase transition due to a breakdown of the quantum liquid nature, i.e., the vanishing linear dispersion for 1D systems. For both WRs, we have the scaling law

$$
R_{\mathrm{W}}^{\kappa, \chi}=\mathcal{F}^{\kappa, \chi}\left[\frac{\left(\eta-\eta_{c}\right)}{T^{\frac{1}{v z}}}\right]+\lambda_{0} T^{-\beta} \mathcal{G}^{\kappa, \chi}\left[\frac{\left(\eta-\eta_{c}\right)}{T^{\frac{1}{v z}}}\right],
$$

where $\beta=(d / z)+1-2 /(v z)$ with $z=2, v=1 / 2$ and $d=1$ for $1 \mathrm{D}$ systems is universal; $\mathcal{F}^{\kappa, \chi}, \mathcal{G}^{\kappa, \chi}$ are the scaling functions (see Appendix C). The second term in (15) with $\beta=-1 / 2$ reflects a contribution from the background at the temperatures above the energy gap $\Delta \sim\left|\eta-\eta_{c}\right|^{z v}$, with driving parameter $\eta$ (e.g., $\mu$ or $H_{r}$ ). These critical exponents agree with the experimental result for the thermal and magnetic properties of the $1 \mathrm{D}$ spin chain at criticality $[19,26]$. For a phase transition from the vacuum to a TLL phase, $\lambda_{0}=0$. Based on (15), the slope of the temperature-rescaled $R_{W}^{\kappa}$ curves at a critical point is given by $\left(\frac{\partial R_{W}^{\kappa}}{\partial \mu}\right)_{\mu_{c}}=C_{r} / T$, see Appendix C.

The significance of the WRs can also be understood from the quantum fluctuations of magnetization, $\left\langle\delta M^{2}\right\rangle=\ell^{d} k_{B} T \chi$, and particle number, $\left\langle\delta N^{2}\right\rangle=\ell^{d} k_{B} T \kappa ; \ell^{d}$ denotes the observation volume in $d$ dimensions. Therefore, microscopically, $\chi$ and $\kappa$ measure the strength of these fluctuations just as $c_{\mathrm{V}}$ quantifies the energy fluctuations; macroscopically, the temperature-independent compressibility and the lineartemperature-dependent specific heat remarkably preserve the nature of quantum liquid at the renormalization fixed point, see Eq. (6) like the Fermi liquid [15]. Consequently, $R_{\mathrm{W}}^{\chi}$ and $R_{\mathrm{W}}^{\kappa}$ characterize the competition between fluctuations of different origin. A constant WR implies that the two types of fluctuations are on an equal footing, regardless of the microscopic details of the underlying many-body system. On the other hand, the growth of the WRs in the critical regime indicates the long-range character of the quantum fluctuations at the quantum phase transitions. 


\section{APPLICATIONS}

\section{A. The Yang-Gaudin model}

In order to show the usefulness of the new ratios, we start by considering the $w=21 \mathrm{D}$ spin-1/2 Fermi gas with a $\delta$-function interaction $[37,38]$. This Yang-Gaudin model has already provided an ideal experimental testing ground [34-36] for few- and many-body physics $[27,39,40]$. For $R_{W}^{\chi}$, the additivity rule of susceptibility and its connection to the $D_{r}^{\chi}$ 's following (12) have been reported in [18]. For $R_{W}^{\kappa}$, we now find from (13) that

$$
R_{W}^{\kappa}=\left(\frac{1}{D_{1}^{\kappa}}+\frac{4}{D_{2}^{\kappa}}\right) /\left(\frac{1}{v_{1}}+\frac{1}{v_{2}}\right) .
$$

Writing the explicit dependence on $\mu, H$, and $g_{1 \mathrm{D}}$ is possible but tedious (we give explicit forms for $D_{1,2}^{\kappa}$ and $v_{1,2}$ in Appendix B). In Fig. 1 we show $R_{W}^{\kappa}$ in the $\mu-H$ plane. $R_{W}^{\kappa}$ elegantly maps out the three finite temperature TLL phases of 1 complexes, i.e., fully-polarized fermions (F), 2 complexes, i.e. pairs $(\mathrm{P})$, and the mixed TLL of excess fermions and pairs $(\mathrm{F}+\mathrm{P})$. The empty vacuum phase $(\mathrm{V})$ is also present in Fig. 1. In particular, we note that for the pure phases $\mathrm{F}$ and $\mathrm{P}, R_{W}^{\kappa} \approx r^{2}$, i.e., close to the strong coupling value $r K_{r}$ [41]. Hence for the Yang-Gaudin model in Fig. 1 we see $R_{\mathrm{W}}^{\kappa}=1$ in phase $\mathrm{F}$ and 4 in phase $\mathrm{P}$. In mixed phases, e.g., F+P, such a constant plateau no longer exists. For $R_{W}^{\kappa}$ in the critical regime, we find a rapid increase due to a strong increase in thermal fluctuations. In Fig. 2 we show the scaling behavior (15) for $R_{W}^{\kappa}$ close to the transition from the vacuum phase into the $\mathrm{F}$ and the $\mathrm{P}$ phases. We find $C_{1} \approx 1.2546$ and $C_{2} \approx 2 \times 5.0185$, respectively. Equation (15) does not contain any free fitting parameter, so one can use this scaling to determine the temperature of the quantum liquid. The scaling
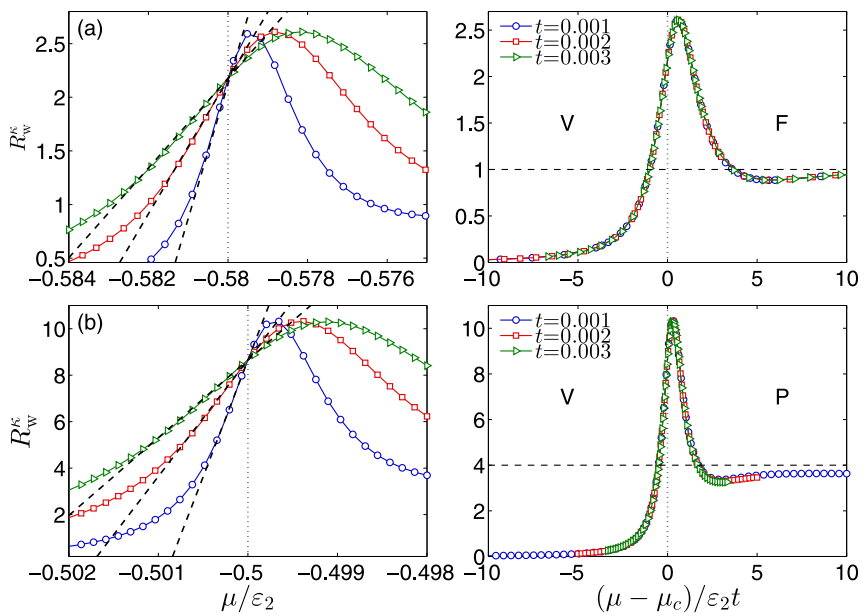

FIG. 2. Scaling of $R_{W}^{\kappa}$ at phase boundaries (a) V-F and (b) V-P with $H / \epsilon_{2}=1.16$ and 0.9 , respectively (cp. Fig. 1). The left panels in (a) and (b) show $R_{W}^{\kappa}$ vs $\mu / \epsilon_{2}$ for reduced temperatures $t=0.003(\triangle)$, $0.002(\square)$, and 0.001 ( $)$. The vertical dotted lines indicate $\mu_{c} / \epsilon_{2}$. The dashed lines show the calculated slopes $C_{r} / t$. The right panels show the scaled $R_{W}^{\kappa}$ as in (15) with $\beta=-1 / 2$ vs $\left(\mu-\mu_{c}\right) / \epsilon_{2} t$. The vertical dotted lines are as in the left panel while the horizontal lines indicate the strong interaction limit of $R_{W}^{\kappa}$ in the (a) F and (b) P phases. law is also similar to the scaling in the non-FL regime of heavy fermions $[3,42]$.

\section{B. A $w \geqslant 3$ quantum liquid}

An even richer phase diagram exists for the threecomponent $\delta$-function interacting Fermi gas with an attractive interaction [31]. Its $T=0$ phases are known to consist of excess fermions $(\mathrm{F})$, pairs $(\mathrm{P})$, trions $(\mathrm{T})$, and mixtures thereof $[43,44]$. Using the TBA equations, we again numerically and analytically calculate the WRs and determine their $H_{1}, H_{2}$, and $T$ dependencies. In Fig. 3 we show that in the pure phases F, P, and T, we have $R_{W}^{\kappa}=1,4,9$, respectively, and the phase boundaries are clearly marked by large increases in $R_{W}^{\kappa}$ near the critical points. At $T>0$, we see that the $\kappa$ and $c_{V}$ curves become progressively more rounded across the phase transitions for $T$ increases. The magnetic field associated with the position of the finite-height peaks varies as a function of temperature. This can be used to define a crossover temperature $T^{*}$ for each such magnetic field corresponding to a peak. For $T<T^{*}$, our analytical and numerical results reconfirm the validity of the additivity rules $(9)-(11)$, i.e., $\kappa$ and $\chi$ remain independent of the temperature and $c_{V}$ depends linearly on the temperature in the TLL phase (cp. lower panel in Fig. 3). This nature is also seen in Fermi liquids $[14,15]$. The additivity nature of the susceptibility is presented in Appendix B. The significance of such additivity rules is the characteristic of quantum liquids at the renormalization fixed point, such as TLLs in integrable models and Fermi liquids in metals, etc.
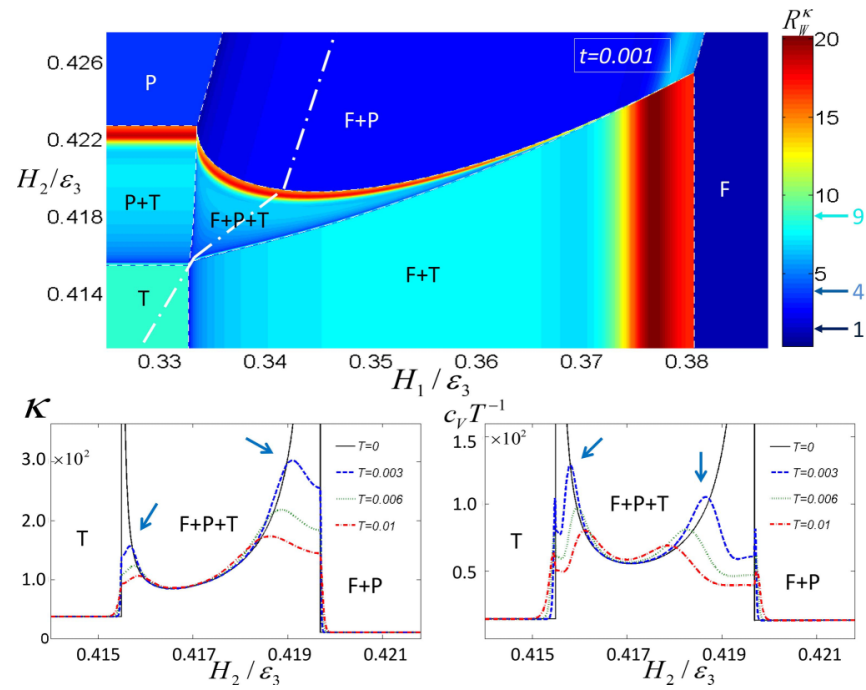

FIG. 3. Upper panel: plot of $R_{W}^{\kappa}$ for the 1D three-component attractive Fermi gas in the $H_{1}-H_{2}$ plane at temperature $t=0.001 \varepsilon_{3} / k_{B}$. The values of $R_{W}^{\kappa}=1,4,9$ in the pure phases F, P, and T, respectively, are indicated by the colors marked on the color scale shown. The dashed lines follow the phase boundaries as indicated. Lower panel: compressibility $\kappa$ and specific heat $c_{V} / T$ vs external field $H_{2} / \varepsilon_{3}$ for a fixed choice of polarization $n_{1}=n_{2}$ indicated by the dash-dotted line in the phase diagram (upper panel). At zero temperature, $\kappa$ and $c_{V} / T$ satisfy the additivity rules (9) and (11) as shown by the solid lines while at finite $T$; they exhibit peaks as indicated by the arrows for $t=0.003 \varepsilon_{3} / k_{B}$. Near the phase boundaries both $\kappa$ and $c_{V} / T$ diverge quickly as $\left(H_{2}-H_{2}^{c}\right)^{-1 / 2}$. 


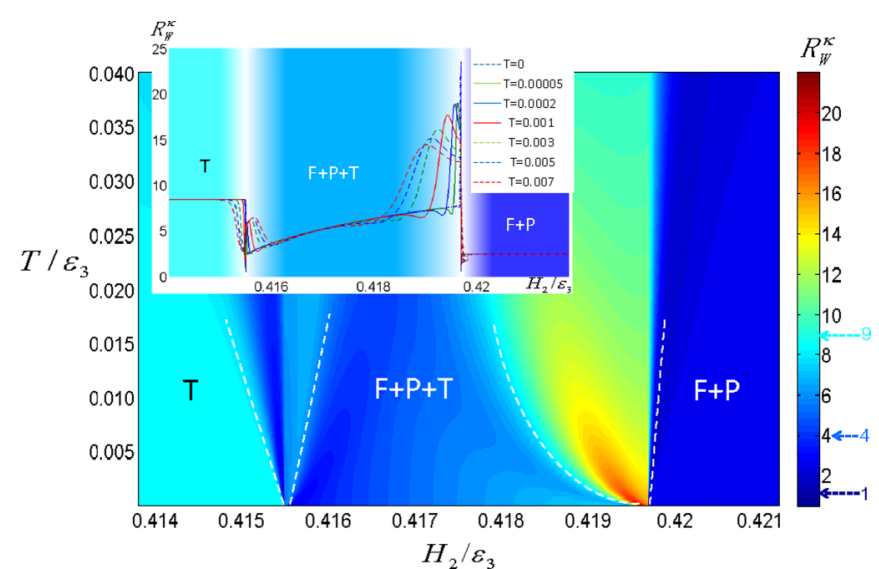

FIG. 4. Mapping out quantum criticality with $R_{W}^{\kappa}$ in the $T-H_{2}$ plane. The $H_{2}$ values have been chosen to follow the fixed polarization $n_{1}=n_{2}$ as indicated by the dash-dotted white line in Fig. 3 . The white dashed lines denote the crossover temperatures $T^{*}$ beyond which the TLL phases break down. The inset shows individual temperature curves of $R_{W}^{\kappa}$ vs $\mathrm{H}_{2}$ for representative temperatures. The bold solid line shows the result corresponding to (13) while the thin dashed lines show the numerical results obtained from the TBA equations (Appendix A).

For $T>T^{*}, R_{W}^{\kappa}$ exhibits the critical scaling behavior (15) (see also Appendix C). Figure 4 shows the contour plot of $R_{W}^{\kappa}$ at low temperatures. The universal quantum critical behavior (15) is characterized by the exponents $z=2, v=1 / 2$. For $T<T^{*}$, the critical exponents are $z=v=1$.

Moreover, using the TBA equations for the $S U(w)$ Fermi gases with an attractive interaction [45], we calculate the susceptibility $\chi_{r}=\partial M / \partial H_{r}$ in response to the change of magnetic field $H_{r}$ for the spin-gapped phases. The other magnetic fields are fixed and the magnetization is given by $M=\sum_{r=1}^{w-1} n_{r} r(w-r) / 2$. In dimensionless units, the energies are rescaled by the interaction energy $\epsilon_{b}=\frac{1}{2} \hbar^{2} c^{2} /(2 m)$, i.e., the temperature $t=T / \epsilon_{b}$, the pressures $\tilde{p}^{r}=p^{r} /\left(|c| \epsilon_{b}\right)$, the susceptibility $\tilde{\chi}=|c| \xi / 2$, see the Appendix B. We obtain explicitly a general expression of the susceptibility $\chi_{r}$ for the gapped phase in which a small number of magnons are created due to the change of $H_{r}$ for $t \ll 1$,

$$
\tilde{\chi}_{r} \approx \frac{1}{4 \sqrt{2 \pi}} \frac{1}{\sqrt{t}} \sqrt{r}(w-r) r e^{-\frac{\Delta r}{t}},
$$

where the gap is given by

$$
\Delta_{r} \approx-r \tilde{\mu}_{r}+\sum_{m=1}^{w} \sum_{\substack{q=1 \\ 2 q \neq r+m}}^{\min (r, m)} \frac{4 \tilde{p}^{m}}{m(r+m-2 q)},
$$

see Appendix C. For example, from the upper panel phase diagram in Fig. 3, we observe that in the gapped phase of $\mathrm{T}$, $\tilde{\chi}_{1}$ with $\Delta_{1}=-\tilde{\mu}_{1}+2 \tilde{p}^{3} / 3$ and $\tilde{\chi}_{2}$ with $\Delta_{2}=-2 \tilde{\mu}_{2}+$ $16 \tilde{p}^{3} / 9$ show a dilute magnon behavior related to the phase transitions from the phase $\mathrm{T}$ into the mixed phase $\mathrm{F}+\mathrm{T}$ and into the the mixed $\mathrm{P}+\mathrm{T}$, respectively. They decay exponentially as the temperature approaches zero. By properly choosing $H_{1}$ and $\mathrm{H}_{2}$, we also can have a phase transition from the gapped phase $\mathrm{T}$ into the mixed phase $\mathrm{F}+\mathrm{P}+\mathrm{T}$.

\section{Critical theory for the $S U(w)$ repulsive Fermi gases}

The existence of internal degrees of freedom significantly changes the quantum magnetism and the dynamics of the system compared to the spinless Bose gas. It is well established that the critical behavior of the spin $S U(w)$ chain can be described by the Wess-Zumino-Witten $\sigma$ model of level $\ell=1$ with the Kac-Moody central charge $C_{s}=\ell\left(w^{2}-1\right) /(\ell+w)$ [46]. The $w$-component repulsive Fermi gases display a $U(1) \otimes S U(w)$ symmetry characterized by one charge degree of freedom and $w-1$ spin rapidities. The low-energy physics of the system is described by the spin-charge separated conformal field theories of an effective Tomonaga-Luttinger liquid and an antiferromagnetic $S U(w)$ Heisenberg spin chain $[5,47]$. By using the TBA equations given in [45] with $H \rightarrow 0$ we find that the pressure of the $1 \mathrm{D}$ repulsive Fermi gases is given by

$$
p=p_{T=0}+\frac{w\left(w^{2}-1\right) H^{2}}{24 \pi v_{\mathrm{s}}}+\frac{\pi T^{2}}{6}\left[\frac{1}{v_{c}}+\frac{C_{s}}{v_{s}}\right],
$$

where $p_{T=0}$ is the pressure at $T=0$ and $v_{s, c}$ are the pseudo Fermi velocities in the spin and charge sectors, respectively. This result is consistent with the critical field theory for the $S U(w)$ spin chains [46]. In the zero magnetic field limit, the spin and charge velocities for the Fermi gas with strong repulsion are, respectively,

$$
\begin{aligned}
& v_{s}=\frac{2 n^{2} \pi^{3} a_{1 \mathrm{D}}}{3 w}\left(1+3 a_{1 \mathrm{D}} Z n\right), \\
& v_{c}=2 n \pi\left(1+2 a_{1 \mathrm{D}} Z n\right),
\end{aligned}
$$

where $Z=-\frac{1}{w}\left[\psi\left(\frac{1}{w}\right)+C\right]$ and $C$ the Euler $\gamma$ constant, and $\psi(x)$ is the Euler $\psi$ function. The susceptibility is then given by Luttinger-liquid relation

$$
\chi v_{s}=\frac{1}{12 \pi} w\left(w^{2}-1\right)
$$

in the limit $H \rightarrow 0$. Indeed the sound velocity $v_{c}$ (20) of the Fermi gas in the large- $w$ limit coincides with that for the spinless Bose gas, whereas $v_{s}$ vanishes quickly as $w$ grows. This gives a reason why the quantum liquid of multicomponent fermions reduces to the liquid of a spinless Bose gas in this limit [34]. In this sense, $R_{W}^{\chi}$ captures this unique large-spin charge separation mechanism in the $w$-component repulsive Fermi gas. Its explicit expression is

$$
R_{W}^{\chi}=\frac{w\left(w^{2}-1\right) v_{c}}{3\left[(w-1) v_{c}+v_{s}\right]},
$$

displaying plateaus of height $w(w+1) / 3$ for either strong repulsion or in the large- $w$ limit, hence capturing the spin degeneracy. For example, $R_{W}^{\chi}=2$ and 4 for the two- and threecomponent Fermi gases with strong repulsion, respectively.

\section{EXPERIMENTAL REALIZATIONS}

Both WRs $R_{W}^{\chi}$ and $R_{W}^{\kappa}$ are readily accessible by experiments. For example, the $1 \mathrm{D} \mathrm{SU}(w) \delta$-function interacting 
Fermi gas of ${ }^{173} \mathrm{Yb}$ has been realized experimentally [34]. It was shown that in the large- $w$ limit the ground state of the gas with a repulsive interaction exhibits properties of a bosonic spinless liquid. In the context of ultracold atoms, it is highly desirable to measure the quantum criticality and the TLL in such Fermi gases with rich spin and charge degrees of freedom. In this scenario, the Yang-Gaudin model is an ideal model to conceive critical phenomena induced from spin and charge interaction effects. This model was recently studied via an ultracold atomic gas in a harmonic trap, such as the two-component ultracold ${ }^{6} \mathrm{Li}$ atoms of Ref. [35].

Due to the harmonic confinement, the chemical potential in the equation of state should be replaced by $\mu(x)=\mu(0)-$ $m \omega^{2} x^{2} / 2$ (within the local density approximation). Here $x$ denotes the position along the 1D trap. Changing $x$ is then equivalent to changing $\mu$, and different phases are located at different spatial positions along the trap. Using a rescaled coordinate $y=x a_{1 \mathrm{D}} /\left(2 a^{2}\right)$ with axial characteristic oscillation length $a=\sqrt{\hbar / m \omega}$, the density profile of the trapped gas can be determined from the dimensionless quantities $N a_{1 \mathrm{D}}^{2} / a^{2}=2 a_{1 \mathrm{D}} \int_{-\infty}^{\infty} d y n(y)$ and polarization $M a_{1 \mathrm{D}}^{2} / a^{2}=$ $2 a_{1 \mathrm{D}} \int_{-\infty}^{\infty} d y m_{z}(y)$. The compressibility can be extracted via
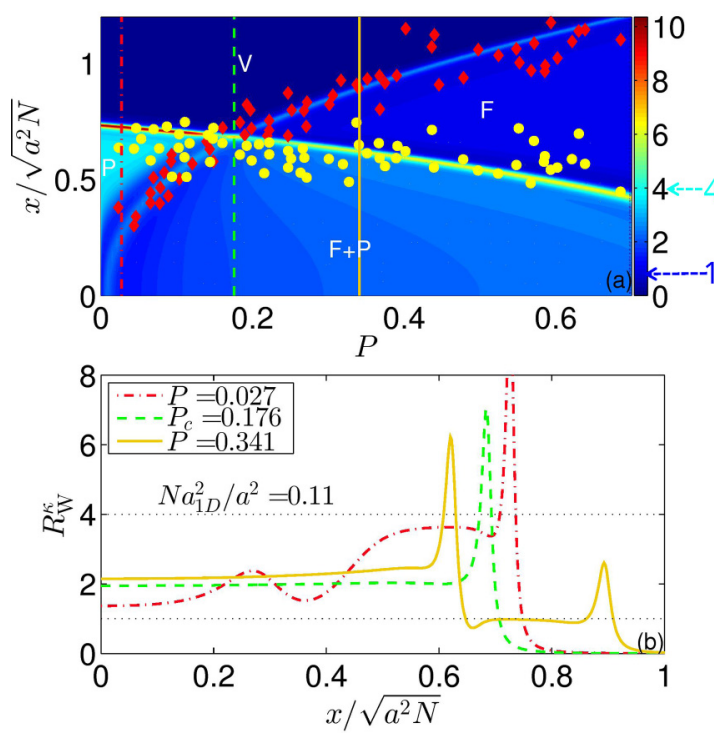

FIG. 5. Upper panel: Contour plot of the WR $R_{W}^{\kappa}$ (2) for the Yang-Gaudin model in a harmonic trap with fixed particle number $N$ and three different polarizations $P=n_{1} / n$ at the temperature $T=0.001 \varepsilon_{2} / k_{B}$. The WR evolves thin round peaks near the phase boundaries, whereas the values of the WR (16) quantify different quantum phases of the TLLs. The WR peaks are in good agreement with the experimental phase boundaries (red and yellow dots) observed in Ref. [35]. Lower panel: the red-dashed and yellow-solid lines show the WR $R_{W}^{\kappa}$ in the trapped gas with the polarizations $P=0.027$ and $P=0.341$, respectively. They indicate segments of the Fulde-Ferrell-Larkin-Ovchinnikov (FFLO) phase in the center accompanied by the wings of the $\mathrm{P}$ and the $\mathrm{F}$ states, respectively. The highest $R_{W}^{\kappa}$ plateau approaches 4 suggesting a quasi-1D superfluid nature, whereas the lowest plateau shows the free fermion nature with $R_{W}^{\kappa}=1$. For a critical polarization $P_{c}=0.176$, the green-dashed line shows that the trapped gas consists solely of the FFLO-like state. $\kappa=\partial n / \partial \mu=-\partial n / \partial x /\left(x m \omega^{2}\right)[48,49]$, while the density $n(x)$ is read off from experimental profiles along $x$. The specific heat can be measured through the sound velocities, while the density waves of pairs and unpaired fermions can be experimentally created by a density depletion or pulse with a far-detuned laser beam [50]. In the upper panel of Fig. 5, we observe that $R_{W}^{\kappa}$ at a very low temperature naturally maps out the phase diagram of the system, showing a good agreement with the experimental phase boundaries [35]. In the lower panel of Fig. 5, we show the behavior for $R_{W}^{\kappa}$, exhibiting the plateau structure as the system passes through different phases upon changing $x$.

\section{CONCLUSIONS}

In summary, we have shown that the additivity rules of physical properties reveal an important characteristic of the TLL and provide the physical origin of the dimensionless WRs. Such dimensionless ratios can be used to identify full TLL phases and capture the essence of phenomena ranging from quantum criticality to spin and charge separation in a wide variety of 1D many-body systems. We have presented some universal relations, such as the WRs (12) and (13), the Luttinger parameter (14), the dimensionless scaling function of the WR (15), the susceptibility for the gapped systems (17), and the WR of $S U(w)$ repulsive Fermi gas related to the level-1 Wess-Zumino-Novikov-Witten conformal theory (22). We also show excellent agreement between the phase diagram predicted by the WRs and the experimentally determined one for the Yang-Gaudin model [35]. Our results therefore successfully demonstrate how to predict universal laws for experimentally realizable quantum liquids in Bose and Fermi degenerate gases, Bose-Fermi mixtures, the 1D Hubbard model, strongly correlated electronic systems, and spin compounds near to and far from quantum critical points on an equal footing.

\section{ACKNOWLEDGMENTS}

We thank T. Guttmann, J. Ho, R. Hulet, G. Shlyapnikov, Z.-C. Yan, S.-Z. Zhang, Q. Zhou, and H. Zhai for helpful discussions. This work has been supported by the National Basic Research Program of China under Grant No. 2012CB922101 and No. 2011CB922200, by the key NNSFC Grant No. 11534014 and by the NNSFC under Grant Nos. 11374331 and 11304357. X.W.G. thanks the Department of Physics at Rice University, University of Washington and ITAMP at Harvard University for kind hospitality. H.Q.L. acknowledges financial support from NSAF U1530401 and computational resources from the Beijing Computational Science Research Centre. R.A.R. gratefully acknowledges the hospitality at WIPM, CAS Wuhan and funding via a CAS Senior Visiting Professorship.

\section{APPENDIX A: THERMODYNAMIC BETHE ANSATZ FOR THE SU $(w)$ FERMI GAS}

The Gibbs free energy of the $\mathrm{SU}(w)$ attractive gas is

$G\left(\mu, H_{1}, \ldots, H_{w}\right)=\sum_{r=1}^{w} \frac{r T}{2 \pi} \int d k \ln \left[1+e^{-\epsilon_{r}(k) / T}\right]$ 
with dispersions $\epsilon_{r}(k)$ defined in the TBA via

$$
\begin{aligned}
\epsilon_{r}(k)= & r k^{2}-r \mu-H_{r}-\varepsilon_{r}+\sum_{p=1}^{r-1}\left\{\sum_{q=p}^{w} a_{q+r-2 p} * T \ln \left[1+e^{-\epsilon_{q}(k) / T}\right]+\sum_{q=r+1}^{w} a_{q-r} * T \ln \left[1+e^{-\epsilon_{q}(k) / T}\right]\right\} \\
& -\sum_{q=1}^{\infty} a_{q} * T \ln \left[1+e^{-\eta_{r, q} / T}\right], \\
\eta_{r, l}(k)= & l \cdot\left(2 H_{r}-H_{r-1}-H_{r+1}\right)+a_{l} * T \ln \left[1+e^{-\epsilon_{r}(k) / T}\right]+\sum_{q=1}^{\infty} U_{l q} * T \ln \left[1+e^{-\eta_{r, q}(k) / T}\right] \\
& \quad-\sum_{q=1} S_{l q} * T \ln \left[1+e^{-\eta_{r-1, q}(k) / T}\right]-\sum_{q=1}^{\infty} S_{l q} * T \ln \left[1+e^{-\eta_{r+1, q}(k) / T}\right]
\end{aligned}
$$

with

$$
\begin{aligned}
& U_{l j}(x)= \begin{cases}a_{|l-j|}(x)+2 a_{|l-j|+2}(x)+\ldots+2 a_{l+j-2}(x)+a_{l+j}(x), & l \neq j \\
2 a_{2}(x)+2 a_{4}(x)+\ldots+2 a_{2 l-2}(x)+a_{2 l}(x), & l=j,\end{cases} \\
& S_{l j}(x)= \begin{cases}a_{|l-j|+1}(x)+2 a_{|l-j|+3}(x)+\ldots+2 a_{l+j-3}(x)+a_{l+j-1}(x), & l \neq j \\
a_{1}(x)+a_{3}(x)+\ldots+a_{2 l-3}(x)+a_{2 l-1}(x), & l=j,\end{cases}
\end{aligned}
$$

while $*$ denotes the convolution $(a * b)(x)=\int a(x-$ $y) b(y) d y$ and the $\eta_{r, l}(k)$ represent the spin string parameters; furthermore $a_{n}(x)=n|c| / 2 \pi\left[(n c / 2)^{2}+x^{2}\right]$. Here $c=$ $m g_{1 \mathrm{D}} / \hbar^{2}=-2 / a_{1 \mathrm{D}}$. The numerical results used in the figures were obtained by solving the above TBA equations.

\section{APPENDIX B: ADDITIVITY RULES FOR SU $(w)$ FERMI GASES}

From (4), we find that constant fields in the grand canonical ensemble, i.e., $d H_{1}=d H_{2}=\ldots=d H_{w-1}=0$, imply $d \mu_{r}=d \mu$ for $r=1, \ldots, w$. Consequently, we have

$$
\kappa=\frac{\partial n}{\partial \mu}=\frac{\partial \sum_{r=1}^{w} r n_{r}}{\partial \mu}=\sum_{r-1}^{w} \frac{r \partial n_{r}}{\partial \mu_{r}}=\sum_{r=1}^{w} \kappa_{r},
$$

with $\kappa_{r}$ as given by (5) and (6). Note that this result is general and does not use special properties of the SU( $w)$ gases.

The additivity rules for the spin susceptibility are very intriguing. For our convenience in analysis of the $\mathrm{SU}(\mathrm{w})$ case, we prefer to keep the ratios among the densities of the charged bound states as $n_{1}: n_{2}: \ldots: n_{w-1}=\lambda_{1}: \lambda_{2}: \ldots: \lambda_{w-1}$, then we can parametrize the $n_{1}, n_{2}, \ldots, n_{w}$ as

$$
n_{r}=\frac{\lambda_{r}}{\lambda} \hat{n},(r=1,2, \ldots, w-1), n_{w}=\frac{1}{w}(n-\hat{n}),
$$

where $\lambda=\lambda_{1}+2 \lambda_{2}+\ldots+(w-1) \lambda_{w-1}$. In order to compute the additivity rules for $\chi_{r}^{-1}=\partial H_{r} / \partial M$, with magnetization $M=\sum_{r=1}^{w-1} r(w-r) n_{r} / 2$, we start with the Legendre transformation from Gibbs free energy to ground state energy: $E=$ $G+\mu n+\sum_{r=1}^{w-1} n_{r} H_{r}$, and in the ensemble $\left\{n_{1}, \cdots, n_{w-1}, n\right\}$ the field $H_{r}$ can be obtained:

$$
\begin{aligned}
H_{r} & =\left.\frac{\partial E}{\partial n_{r}}\right|_{n_{1}, \cdots, n_{r-1}, n_{r+1}, \cdots, n_{w-1}, n}-\left.\frac{r}{w} \frac{\partial E}{\partial n_{w}}\right|_{n_{1}, \cdots, n_{w-1}}, \\
& =\left.\frac{\partial E}{\partial n_{r}}\right|_{n_{1}, \cdots, n_{r-1}, n_{r+1}, \cdots, n_{w}},
\end{aligned}
$$

where $E=\sum_{r=1}^{w} E_{r}$ denotes the energy of the multicomponent ground state with a subtraction of the binding energies [27] and we have $\partial E / \partial n_{r}=r \mu_{r}$ for consistency with (4). We emphasize that the additivity of $E$ is a fundamental property of a TLL, implied by the linearity of the dispersions [27].

Following the relation (B2), we can define differential forms of any thermodynamic function $f=f\left(n_{1}, \cdots, n_{w}\right)$ :

$$
\begin{gathered}
d f=\sum_{r=1}^{w-1} \frac{\partial f}{\partial n_{r}} \cdot \frac{\lambda_{r}}{\lambda} \Delta \hat{n}-\frac{\partial f}{\partial n_{w}} \frac{1}{w} \Delta \hat{n}, \\
d M=\sum_{r=1}^{w-1} \frac{1}{2} r(w-r) \frac{\lambda_{r}}{\lambda} \cdot \Delta \hat{n} .
\end{gathered}
$$

We denote the operator $\mathcal{D}_{r}=\frac{\partial}{\partial n_{r}}-\frac{r}{w} \cdot \frac{\partial}{\partial n_{w}}$ with $r=$ $1,2, \ldots, w-1$. Then the field $H_{r}$ can be expressed explicitly: $H_{r}=\mathcal{D}_{r} E$ and the susceptibility in response to the $H_{r}$ can be expressed as

$$
\frac{1}{\chi_{r}}=\frac{d H_{r}}{d M}=\frac{\sum_{r=1}^{w-1} \lambda_{r} \mathcal{D}_{r}}{\sum_{r=1}^{w-1} \frac{1}{2} r(w-r) \lambda_{r}} \cdot \mathcal{D}_{r} E .
$$

We note that the last term in the Eq. (B3) linearly depends on the density $n_{r}$ and $n_{w}$. Therefore it can be safely dropped off in the calculation of the susceptibilities because of the second order of derivatives. In terms of the ground state energies $E=\sum_{\ell=1}^{w} E_{\ell}$ for the individual charge bound states, we define the stiffness as

$$
\frac{1}{\chi_{r \ell}}=\frac{\hbar \pi}{r^{2}} D_{r, l}^{\chi}=\frac{\sum_{r=1}^{w-1} \lambda_{r} \mathcal{D}_{r}}{\sum_{r=1}^{w-1} \frac{1}{2} r(w-r) \lambda_{r}} \cdot \mathcal{D}_{r} E_{\ell},
$$

where $r=1,2, \ldots, w-1$ and $\ell=1,2, \ldots, w$. Consequently, we have the susceptibility (10) in response to the external field $H_{r}$. 

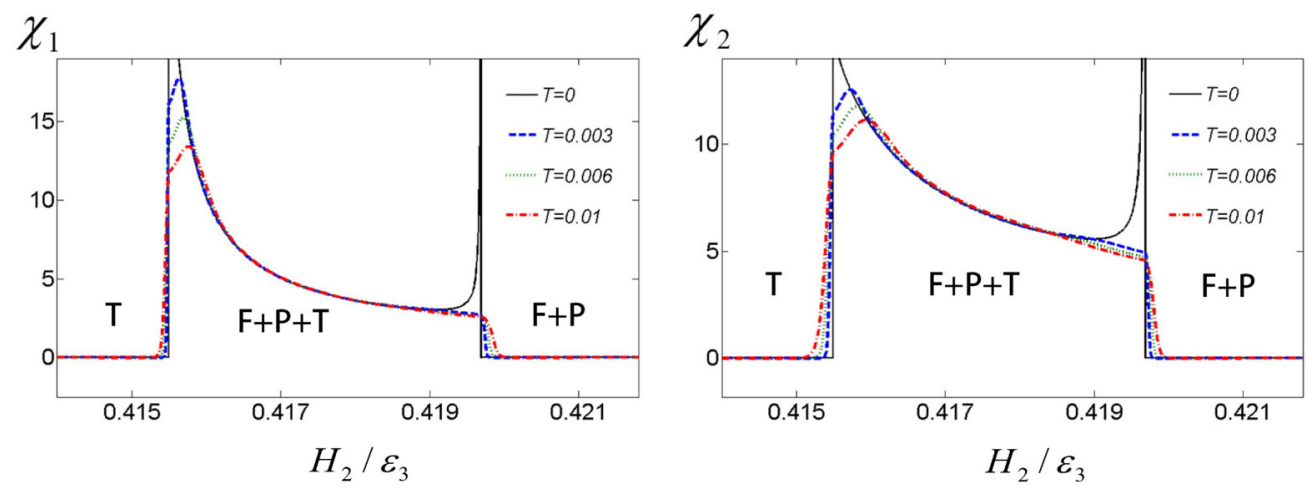

FIG. 6. Susceptibilities in response to $H_{1}$ and $H_{2}$ for the three-component Fermi gas with a fixed ratio of $n_{1} / n_{2}=1$ at finite temperatures. Under this setting the $H_{1}$ and $H_{2}$ have a one-to-one mapping along the line of $n_{1} / n_{2}=1$ with a fixed total density. These two figures show that the susceptibility is temperature independent for the TLL phase of $\mathrm{F}+\mathrm{P}+\mathrm{T}$ indicated by the long dashed line in Fig. 3 in the main text. The deviation from the solid lines in the two figures shows a breakdown of the TLL. Here T, B, and F stand for trions, pairs, and excess fermions, respectively. The solid lines confirm the additivity rule Eq. (10) in the main text.

In fact, Zeeman splitting can be characterized by the Zeeman energy levels $\epsilon_{Z}^{r}$ or by the effective magnetic fields $H_{r}$ with $r=1,2, \ldots, w$. Here $H_{w}=0$. Both sets of parameters are related via the relation

$$
\sum_{r=1}^{w} \epsilon_{Z}^{r} n^{r}=-\sum_{r=1}^{w} H_{r}\left(n^{r}-n^{r+1}\right)
$$

A consistent solution of this equation gives the relations between $H_{r}$ and Zeeman energy levels [45]. If we denote the difference between the energy levels of fermions in the states $|m+1\rangle$ and $|m\rangle$ as $\Delta_{m+1, m}=\epsilon_{Z}^{m+1}-\epsilon_{Z}^{m}$, then the total susceptibility $\chi=\frac{d M}{d \Delta_{\text {total }}}$ with $\Delta_{\text {total }}=\sum_{r=1}^{w-1} \Delta_{r+1, r}$ is given by

$$
\begin{aligned}
& \chi^{-1}= \frac{d \sum_{r=1}^{w-1} \Delta_{r+1, r}}{d M}=\frac{d\left(H_{1}+H_{w-1}\right)}{d M} \\
&=\frac{1}{\chi_{1}}+\frac{1}{\chi_{w-1}} .
\end{aligned}
$$

Using the numerical calculation for the Gibbs free energy $G\left(\mu, H_{1}, H_{2}\right)$ from Eq. (A1), we observe that the susceptibilities $\chi_{1}$ and $\chi_{2}$ satisfy the relation (10) in the TLL phase of trions, pairs, and single atoms, see Fig. 6 .

Using the TBA equations [28,45], one can easily prove that the specific heat can be written in terms of sound velocities, i.e.,

$$
c_{V}=\frac{\pi^{2} k_{\mathrm{B}}^{2} T}{3} \sum_{r}^{w} \frac{1}{\hbar \pi v_{r}} .
$$

\section{APPENDIX C: SCALING FORMS ON BOUNDARIES}

We focus on the low temperature behavior for $w=3$ to derive the analytical results for the thermodynamics, and finally we derive a formula of general $\mathrm{SU}(w)$ gases of the criticality about susceptibilities. In the strong interaction limit, we can solve these TBA equations analytically at low temperatures $T \ll \epsilon_{3} / k_{\mathrm{B}}$ by simplifying

$$
\varepsilon_{r}(k) \approx r k^{2}-A_{r}, \quad r=1,2,3,
$$

with

$$
\begin{aligned}
A_{1}= & \mu+H_{1}-\frac{2}{|c|} p_{2}-\frac{2}{3|c|} p_{3}+\frac{1}{4|c|^{3}} Y_{2, \frac{5}{2}} \\
& +\frac{1}{9|c|^{3}} Y_{3, \frac{5}{2}}+T e^{-\left(2 H_{1}-H_{2}\right) / T} e^{-J_{1} / T} I_{0}\left(J_{1} / T\right) \\
A_{2}= & 2 \mu+\frac{1}{2} c^{2}+H_{2}-\frac{4}{|c|} p_{1}-\frac{1}{|c|} p_{2}-\frac{16}{9|c|} p_{3} \\
& +\frac{8}{|c|^{3}} Y_{1, \frac{5}{2}}+\frac{1}{4|c|^{3}} Y_{2, \frac{5}{2}}+\frac{224}{243|c|^{3}} Y_{3, \frac{5}{2}} \\
& +T e^{-\left(2 H_{2}-H_{1}\right) / T} e^{-J_{2} / T} I_{0}\left(J_{2} / T\right), \\
A_{3}= & 3 \mu+2 c^{2}-\frac{2}{|c|} p_{1}-\frac{8}{3|c|} p_{2}-\frac{1}{|c|^{2}} p_{3} \\
& +\frac{1}{2|c|^{3}} Y_{1, \frac{5}{2}}+\frac{28}{27|c|^{3}} Y_{2, \frac{5}{2}}+\frac{1}{16|c|^{3}} Y_{3, \frac{5}{2}}
\end{aligned}
$$

with $Y_{r, a}=-\sqrt{\frac{r}{4 \pi}} T^{a} \operatorname{Li}_{a}\left(-e^{A_{r} / T}\right)$. Here the effective spin coupling constant is $J_{r}=2 p_{r} / r|c|$ with $c=m g_{1 D} / \hbar^{2}$. The polylogarithm function is defined as $\operatorname{Li}_{n}(x)=\sum_{k=1}^{\infty} \frac{x^{k}}{k^{n}}$ and $I_{0}(x)=\sum_{k=0}^{\infty} \frac{1}{(k !)^{2}}(x / 2)^{2 k}$. The effective pressures $p_{r}$, with $r=1,2,3$, of excess fermions, pairs, and trions, respectively, can be expressed as

$$
\begin{aligned}
& p_{1}=Y_{1, \frac{3}{2}}\left[1+\frac{4}{|c|^{3}} Y_{2, \frac{3}{2}}+\frac{1}{3|c|^{3}} Y_{3, \frac{3}{2}}\right], \\
& p_{2}=Y_{2, \frac{3}{2}}\left[1+\frac{4}{|c|^{3}} Y_{1, \frac{3}{2}}+\frac{1}{4|c|^{3}} Y_{2, \frac{3}{2}}+\frac{112}{81|c|^{3}} Y_{3, \frac{3}{2}}\right], \\
& p_{3}=Y_{2, \frac{3}{2}}\left[1+\frac{1}{3|c|^{3}} Y_{1, \frac{3}{2}}+\frac{112}{81|c|^{3}} Y_{2, \frac{3}{2}}+\frac{1}{8|c|^{3}} Y_{3, \frac{3}{2}}\right] .
\end{aligned}
$$

\section{Vacuum-Pair}

The above simplified TBA equations (C1) can be used to derive universal low temperature properties of the $\mathrm{SU}(2)$ and SU(3) Fermi gases. In the following, we derive the scaling forms of the Wilson ratios in the critical regions for the twocomponent Fermi gas. Here we will use the dimensionless units as explained in the main text. The phase boundary for the phase 
transition from $\mathrm{V}$ to $\mathrm{P}$ phase is $\tilde{\mu}_{c}=-\frac{1}{2}$ for $h=H / \varepsilon_{2}<1$. Near this critical point, the scaling forms of specific heat, compressibility, and susceptibility in dimensionless units are given by

$$
\begin{gathered}
\frac{c_{V}}{|c| t} \approx-\frac{2}{\sqrt{\pi t}} \mathcal{G}\left(\frac{2\left(\tilde{\mu}-\tilde{\mu}_{c}\right)}{t}\right), \\
\tilde{\kappa} \approx-\frac{2}{\sqrt{\pi t}} \mathcal{F}\left(\frac{2\left(\tilde{\mu}-\tilde{\mu}_{c}\right)}{t}\right), \\
\tilde{\chi} \approx 0 .
\end{gathered}
$$

where the functions $\mathcal{G}(x)=\frac{3}{16} \operatorname{Li}_{\frac{3}{2}}\left(-e^{x}\right)-\frac{1}{4} x \operatorname{Li}_{\frac{1}{2}}\left(-e^{x}\right)+$ $\frac{1}{4} x^{2} \mathrm{Li}_{-\frac{1}{2}}\left(-e^{x}\right)$ and $\mathcal{F}(x)=\mathrm{Li}_{-\frac{1}{2}}\left(-e^{x}\right)$.

\section{Vacuum-Fully polarized}

The phase boundary for the phase transition from $\mathrm{V}$ to $\mathrm{F}$ phase is $\tilde{\mu}_{c}=-\frac{h}{2}$, for $h>1$; the scaling forms in dimensionless units are

$$
\begin{aligned}
\frac{c_{V}}{|c| t} & \approx-\frac{1}{2 \sqrt{2 \pi t}} \mathcal{H}\left(\frac{\tilde{\mu}-\tilde{\mu}_{c}}{t}\right), \\
\tilde{\kappa} & \approx-\frac{1}{2 \sqrt{2 \pi t}} \mathcal{F}\left(\frac{\tilde{\mu}-\tilde{\mu}_{c}}{t}\right), \\
\tilde{\chi} & \approx-\frac{1}{8 \sqrt{2 \pi t}} \mathcal{F}\left(\frac{\tilde{\mu}-\tilde{\mu}_{c}}{t}\right),
\end{aligned}
$$

where $\mathcal{H}(x)=\frac{3}{4} \operatorname{Li}_{\frac{3}{2}}\left(-e^{x}\right)-x \operatorname{Li}_{\frac{1}{2}}\left(-e^{x}\right)+x^{2} \operatorname{Li}_{-\frac{1}{2}}\left(-e^{x}\right)$.

\section{Pair-Partially polarized}

The critical fields for the phase transition from $\mathrm{P}$ to $\mathrm{F}+\mathrm{P}$ phase are $\tilde{\mu}_{c}=-\frac{h}{2}+\frac{4}{3 \pi}(1-h)^{\frac{3}{2}}$, for $h<1$; the scaling forms are

$$
\begin{gathered}
\frac{c_{V}}{|c| t} \approx-\frac{1}{2 \sqrt{2 \pi t}} \mathcal{R}\left(\frac{\tilde{\mu}-\tilde{\mu}_{c}}{t}\right), \\
\tilde{\kappa} \approx \kappa_{o 1}-\frac{\lambda_{1}}{2 \sqrt{2 \pi t}} \mathcal{F}\left(\frac{\tilde{\mu}-\tilde{\mu}_{c}}{t}\right), \\
\tilde{\chi} \approx-\frac{\lambda_{2}}{8 \sqrt{2 \pi t}} \mathcal{F}\left(\frac{\tilde{\mu}-\tilde{\mu}_{c}}{t}\right),
\end{gathered}
$$

where $b=(1-h)\left(1+\frac{2}{\pi} \sqrt{1-h}\right), \lambda_{1}=1+\frac{2 \sqrt{b}}{\pi}-\frac{10 b}{\pi^{2}}, \lambda_{2}=$ $1-\frac{3 \sqrt{b}}{\pi}+\frac{6 b}{\pi^{2}}, \quad \kappa_{o 1}=\frac{2}{\pi \sqrt{b}} \lambda_{1}, \quad$ and $\quad \mathcal{R}(x)=\frac{3}{4} \operatorname{Li}_{\frac{3}{2}}\left(-e^{x}\right)-$ $x \operatorname{Li}_{\frac{1}{2}}\left(-e^{x}\right)+x^{2} \operatorname{Li}_{-\frac{1}{2}}\left(-e^{x}\right)$.

\section{Fully-Partially polarized}

For the phase transition from $\mathrm{F}$ to $\mathrm{F}+\mathrm{P}$ phase, we have $\tilde{\mu}_{c}=-\frac{1}{2}+\frac{1}{3 \pi}(h-1)^{\frac{3}{2}}, h>1$; with scaling forms

$$
\begin{gathered}
\frac{c_{V}}{|c| t} \approx-\frac{1}{2 \sqrt{2 \pi t}} \mathcal{S}\left(\frac{2\left(\tilde{\mu}-\tilde{\mu}_{c}\right)}{t}\right), \\
\tilde{\kappa} \approx \kappa_{o 3}-\frac{\lambda_{3}}{2 \sqrt{2 \pi t}} \mathcal{F}\left(\frac{2\left(\tilde{\mu}-\tilde{\mu}_{c}\right)}{t}\right), \\
\tilde{\chi} \approx \chi_{o 4}-\frac{\lambda_{4}}{8 \sqrt{2 \pi t}} \mathcal{F}\left(\frac{2\left(\tilde{\mu}-\tilde{\mu}_{c}\right)}{t}\right) .
\end{gathered}
$$

where the constants are given by $a=(h-1)\left(1+\frac{2}{3 \pi} \sqrt{h-1}\right)$, $\lambda_{3}=4 \sqrt{2}\left(1+\frac{\sqrt{a}}{\sqrt{2} \pi}-\frac{a}{\pi^{2}}\right), \quad \lambda_{4}=\frac{8 \sqrt{2} a}{\pi^{2}}, \quad \kappa_{o 3}=\frac{1}{2 \sqrt{2} \pi \sqrt{a}}, \quad$ and $\chi_{o 4}=\frac{1}{8 \sqrt{2} \pi \sqrt{a}}\left(1-\frac{3 \sqrt{a}}{\pi}\right)$. The dimensionless function is given by $S(x)=\frac{3}{2 \sqrt{2}} \operatorname{Li}_{\frac{3}{2}}\left(-e^{x}\right)-\sqrt{2} x \operatorname{Li}_{\frac{1}{2}}\left(-e^{x}\right)+$ $\sqrt{2} x^{2} \mathrm{Li}_{-\frac{1}{2}}\left(-e^{x}\right)$. The slopes of the Wilson ratio curves at the critical point $\mu=\mu_{c}$ reveal a unique temperature-dependent feature, namely the slope at the critical point, $\left(\frac{\partial R_{\mathrm{w}}^{\kappa}}{\partial \mu}\right)_{\mu_{c}} \equiv \frac{C_{r}}{T}$, is given as

$$
c_{r}=\frac{r \pi^{2}}{3} \frac{\left(\mathcal{F}^{\prime}(0) \mathcal{G}(0)-\mathcal{F}(0) \mathcal{G}^{\prime}(0)\right)}{\mathcal{G}(0)^{2}},
$$

i.e., is a constant for the phase transition from vacuum into an $r$-complex TLL phase.

\section{Susceptibilities for the gapped phase in the attractive $S U(w)$ gases}

The total polarization of $S U(w)$ gas can be expressed as $\tilde{m}=\sum_{r=1}^{w} \frac{1}{2} \tilde{n}_{r} r(w-r)$, then we can obtain the susceptibility:

$$
\tilde{\chi}_{r}=\frac{\partial \tilde{m}}{\partial \tilde{h}_{r}}=\sum_{k, l=1}^{w} \frac{1}{2} k(w-k) \frac{\partial^{2} \tilde{p}^{(l)}}{\partial \tilde{h}_{k} \partial \tilde{h}_{r}} ;
$$

in the limit of $t \rightarrow 0$ and $|c| \gg 1$, the leading behavior of the second order derivatives of pressures reads

$$
\frac{\partial^{2} \tilde{p}^{l}}{\partial \tilde{h}_{k} \partial \tilde{h}_{r}} \approx-\frac{\sqrt{r}}{2} \frac{t^{-\frac{1}{2}}}{\sqrt{\pi}} \mathrm{Li}_{-\frac{1}{2}}\left(-\mathrm{e}^{\frac{\tilde{\AA}^{(\mathrm{r})}}{\mathrm{t}}}\right) \delta_{1, \mathrm{k}} \delta_{1, \mathrm{r}} .
$$

Substituting (C17) into (C16), we arrive at the explicit form of the susceptibility $\tilde{\chi}_{r}$ corresponding to the field $H_{r}$ when the other fields are fixed

$$
\begin{aligned}
\tilde{\chi}_{r} & \approx-\frac{t^{-\frac{1}{2}}}{4 \sqrt{2 \pi}} \sqrt{r}(w-r) r \mathrm{Li}_{-\frac{1}{2}}\left(-\mathrm{e}^{\frac{\tilde{\tilde{A}}^{(\mathrm{r})}}{\mathrm{t}}}\right) \\
& \approx \frac{t^{-\frac{1}{2}}}{4 \sqrt{2 \pi}} \sqrt{r}(w-r) r e^{-\frac{\Delta_{r}}{t}},
\end{aligned}
$$

where the gap $\Delta_{r}$ is related to the effective chemical potential through $\Delta_{r}=-\tilde{A}^{(r)}$, which can be determined from the TBA equations

$$
\tilde{A}^{(r)}=r \tilde{\mu}-\sum_{m=1}^{w} \sum_{\substack{q=1 \\ 2 q \neq r+m}}^{\min (r, m)} \frac{4 \tilde{p}^{m}}{m(r+m-2 q)} .
$$

Here the second approximately equal in (C18) holds when $\Delta_{r}>0$, which implies the existence of the gap and the susceptibility presents exponential decay, otherwise $\tilde{\chi}_{r} \approx$ $\frac{\sqrt{r}(w-r) r}{4 \sqrt{2} \pi \sqrt{-\Delta_{r}}}$, which is a positive constant when $t \rightarrow 0$. Note that the result in $(\mathrm{C} 18)$ is different by a factor $\frac{1}{2}$ due to the convention of $H_{1}=H / 2$ in the SU(2) case.

\section{APPENDIX D: EXPLICIT FORMULAS FOR THE SU(2) FERMI GAS}

In the mixed phase for $\mathrm{SU}(2)$ Fermi gas with one magnetic field $H_{1}$, we have $n=2 n_{2}+n_{1}$ and $\chi_{1,1}=\left(\mu_{\mathrm{B}} \mathfrak{g}\right)^{2}\left(\partial n_{1} / \partial \mu_{1}\right)_{n}, \quad \chi_{1,2}=2\left(\mu_{\mathrm{B}} \mathfrak{g}\right)^{2}\left(\partial n_{2} / \partial \mu_{2}\right)_{n}$, with 
the stiffnesses in canonical ensemble $D_{1}^{\chi}=\frac{1}{\hbar \pi}\left(\partial \mu_{1} / \partial n_{1}\right)_{n}$, $D_{2}^{\chi}=\frac{2}{\hbar \pi}\left(\partial \mu_{2} / \partial n_{2}\right)_{n} . \quad$ Similarly, $\quad \kappa_{1}=\left(\partial n_{1} / \partial \mu_{1}\right)_{H}$ and $\quad \kappa_{2}=2\left(\partial n_{2} / \partial \mu_{2}\right)_{H} \quad$ with $\quad D_{2}^{\kappa}=\frac{2}{\hbar \pi}\left(\partial \mu_{2} / \partial n_{2}\right)_{H}$, $D_{1}^{\kappa}=\frac{1}{\hbar \pi}\left(\partial \mu_{1} / \partial n_{1}\right)_{H}$ defined in the grand canonical ensemble.

For strong coupling, we find the explicit form of the $\mu_{r}$ in terms of the densities of pairs $n_{2}$ and unpaired fermions $n_{1}$ (in units of $\left.\hbar^{2} /(2 m)\right)$ [45] to be

$$
\begin{aligned}
\mu_{2} \approx & \pi^{2}\left(\frac{n_{2}^{2}}{4}+\frac{2 n_{2}^{3}}{3|c|}+\frac{n_{2}^{2} n_{1}}{|c|}+\frac{4 n_{1}^{3}}{3|c|}+\frac{3 n_{1}^{2} n_{2}^{2}}{c^{2}}+\frac{5 n_{2}^{4}}{4 c^{2}}\right. \\
& \left.+\frac{4 n_{1} n_{2}^{3}}{c^{2}}+\frac{16 n_{1}^{3} n_{2}}{c^{2}}\right), \\
\mu_{1} \approx & \pi^{2}\left(n_{1}^{2}+\frac{8 n_{1}^{2} n_{2}}{|c|}+\frac{2 n_{2}^{3}}{3|c|}+\frac{48 n_{1}^{2} n_{2}^{2}}{c^{2}}+\frac{4 n_{2}^{3} n_{1}}{c^{2}}+\frac{2 n_{2}^{4}}{c^{2}}\right) .
\end{aligned}
$$

Here $c=m g_{1 \mathrm{D}} / \hbar^{2}=-2 / a_{1 \mathrm{D}}$ is the interaction strength. Therefore, the compressibilities and susceptibilities are given by [in units of $\left.\hbar^{2} /(2 m)\right]$, e.g.,

$$
\begin{aligned}
& \kappa_{2}^{-1} \approx \frac{\pi^{2} n_{2}}{4}\left(1+\frac{6 n_{1}}{|c|}+\frac{4 n_{2}}{|c|}+\frac{n_{2}^{2}}{2|c| n_{1}}+\frac{24 n_{1}^{2}}{c^{2}}\right. \\
&\left.+\frac{24 n_{1} n_{2}}{c^{2}}+\frac{17 n_{2}^{2}}{c^{2}}-\frac{2 n_{2}^{3}}{c^{2} n_{1}}+\frac{n_{2}^{4}}{4 c^{2} n_{1}^{2}}\right), \\
& \kappa_{1}^{-1} \approx 2 \pi^{2} n_{1}\left(1+\frac{12 n_{2}}{|c|}+\frac{16 n_{1}^{2}}{|c| n_{2}}+\frac{96 n_{2}^{2}}{c^{2}}+\frac{384 n_{1}^{2}}{c^{2}}\right. \\
&\left.-\frac{8 n_{1} n_{2}}{c^{2}}-\frac{96 n_{1}^{3}}{c^{2} n_{2}}+\frac{256 n_{1}^{4}}{c^{2} n_{2}}\right) \\
& \chi_{1,1}^{-1}=\pi^{2} n_{2}\left[1+\frac{4}{|c|}\left(n-3 n_{2}\right)+\frac{3}{c^{2}}\left(4 n^{2}-24 n n_{2}+30 n_{2}^{2}\right)\right] \text { (D5) } \\
& \chi_{1,2}^{-1}=8 \pi^{2} n_{1}\left[1+\frac{4}{|c|}\left(n-2 n_{1}\right)+\frac{4}{c^{2}}\left(2 n^{2}+10 n_{1}^{2}-12 n n_{1}\right)\right] .
\end{aligned}
$$

In the above calculation for compressibility, the condition $H=$ $2\left(\mu_{1}-\mu_{2}\right)+c^{2} / 4$ was used, i.e., $d H=0$ gives (up to the $O\left(\frac{1}{c^{2}}\right)$ order $)$ :

$$
\frac{d n_{1}}{d n_{2}}=\frac{n_{2}}{4 n_{1}}\left(1-\frac{8 n_{2}}{|c|}+\frac{6 n_{1}}{|c|}+\frac{n_{2}^{2}}{2|c| n_{1}}-\frac{16 n_{1}^{2}}{|c| n_{2}}\right) .
$$

Moreover, the interaction effect enters into the collective velocities $v_{1}, v_{2}$ of the excess fermions and bound pairs. For strong attraction, they are given by [27]

$$
\begin{aligned}
& v_{1} \approx \frac{\hbar}{2 m} 2 \pi n_{1}\left(1+8 n_{2} /|c|+48 n_{2}^{2} / c^{2}\right), \\
& v_{2} \approx \frac{\hbar}{2 m} \pi n_{2}\left(1+2 A /|c|+3 A^{2} / c^{2}\right),
\end{aligned}
$$

with $A=2 n_{1}+n_{2}$.

\section{APPENDIX E: CONNECTION BETWEEN THE LUTTINGER AND FERMI LIQUIDS}

\section{Wilson Ratios}

Now we further build up a connection between the TLL and the Fermi liquid. By definition (1) and (2), the two type of Wilson ratios of interacting Fermi liquid in 3D are given by

$$
R_{\mathrm{W}}^{\chi}=\frac{1}{1+F_{0}^{a}}, \quad R_{\mathrm{W}}^{\kappa}=\frac{1}{1+F_{0}^{s}},
$$

which depends on Landau parameters $F_{0}^{a, s}$ characterizing the interaction [3]. This is very similar to our finding for 1D systems, see Eqs. (12) and (13). However, it's extremely hard to calculate the Landau parameters $F_{0,1}^{s, a}$ in Fermi liquid theory due to the reason that the density of state $N^{*}(0)$ cannot be obtained explicitly. Fermi liquid theory elegantly maps an interacting system into a free fermion system where the interaction is encoded into the density of state and effective mass. But the Fermi liquid theory is not valid in $1 \mathrm{D}$ interacting systems because there does not exist a well-defined quasiparticle. Here we demonstrate that exact solution of the TBA equations does show a novel existence of Fermi-liquid-like signature in 1D interacting systems. In fact, the additivity rules which we found in previous sections reflect a 1D Fermi-liquid-like nature, also see a discussion in Ref. [16].

\section{Feedback interaction}

The excitations near Fermi points in 1D many-body systems can form a collective motion independent of microscopic details of systems, namely, there exists a certain dispersion relation between energy and momentum. We observe that integrable systems provide a deep understanding of the intrinsic connection between the TLL and the Fermi liquid. In fact, the TBA equations of 1D systems give the exact dressed energies and determine the dispersion relations of each branch. At low temperatures, only the behavior of the dressed energies near the zero point, or say, the $k_{F}$ and $v_{F}$ at the $1 \mathrm{D}$ Fermi points, determines the first order and second orders of thermodynamic quantities. In general, for the attractive $S U(N)$ Fermi gases, the spin fluctuations are suppressed. Therefore the TBA equations can be rewritten as

$$
\begin{aligned}
\varepsilon^{(r)}(k) & =\epsilon_{r}^{0}(k)-\sum_{s=1}^{N} A_{r s} * \varepsilon_{-}^{(s)}(k), \quad(r=1,2, \cdots, w), \\
A_{m n} & =\sum_{q=1}^{\min (m, n)} a_{m+n-q},
\end{aligned}
$$

where $\epsilon_{r}^{0}=\frac{\hbar^{2}}{2 m} r\left(k^{2}-\mu_{r}\right)=\frac{p_{0}^{2}}{2 r m}-\frac{\hbar^{2}}{2 m} r \mu_{r}$ is the first order coefficient describing excitation energy of a single $r$ complex; here $\mu_{r}$ is the effective chemical potential. Here $p_{0}=r \hbar k$. The function $a_{m}(x)$ is defined in Appendix A. These equations show a similar form of 'feedback interaction' equation in the Landau Fermi liquid theory $[15,16]$. This encourages us to further find a perturbation idea into our calculations. Below we give a Fermi-liquid-like description for the low energies of the 1D attractive Fermi gases, i.e., mapping an interacting system with multisubsystems to a multicomponent free system. 


\section{Phenomenological description}

The symmetry group of the interaction of quasiparticles in the 1D "Fermi liquid" is reduced from the $S O$ (3) group to the cyclic group $C_{2}$. Therefore, interaction parameter between the 'quasi' momentum $p$ and $p$ ' could be written as $f_{p, p^{\prime}}^{s, a}=f_{0}^{s, a}+f_{1}^{s, a} \operatorname{sign}\left(p \cdot p^{\prime}\right)$. Then following the conventional Fermi liquid theory, we still can have Landau parameters $F_{0,1}^{s, a}$, which are interacting parameters [16] and describe the main properties of our system. Conventional sound velocity is associated with oscillations in the density of a fluid, and hydrodynamics gives

$$
v^{2}=\frac{\kappa}{\rho}=\frac{\kappa}{m n}
$$

where $\rho=m n$ is the density of the fluid and $\kappa=-L \frac{\partial P}{\partial L}$ is the bulk modulus. In FL theory $\kappa=\frac{n^{2}}{N^{*}(0)}\left(1+F_{0}^{s}\right)$, where the $N^{*}(0)$ denotes the density of state in momentum space near the Fermi surface, therefore the velocity of first sound is given by

$$
v^{2}=\frac{n}{m N^{*}(0)}\left(1+F_{0}^{s}\right)
$$

By the 1D analog of Fermi liquid theory, we have the relations [16] $n=\frac{p_{F}}{\pi}, N^{*}(0)=\frac{1}{\pi} \frac{m^{*}}{p_{F}}$ and $m=m^{*}\left(1+F_{1}^{s}\right)$. Then we obtain:

$$
v^{2}=v_{F}^{2}\left(1+F_{0}^{s}\right)\left(1+F_{1}^{s}\right)
$$

Here, due to the collective motion, the backflow does not exist in $1 \mathrm{D}$ quantum liquids, therefore $F_{1}^{s} \approx 0$. This can be seen from the dressed energy equation (E2), where the effective mass $r m$ is almost unchanged up to the order of $O\left(c^{-2}\right)$ for a strong coupling regime.

\section{Consistency}

Using the exact solutions, we can calculate the Wilson ratio $R_{W}^{c}$ for different phases of TLLs. For a single state of an $r$ complex, the Wilson ratio is known from Eq. (E1). Then the parameter $F_{0,1}^{s}$ could be determined by a comparison with the Bethe ansatz result in the strong coupling limit (i.e., $|c| \rightarrow \infty$ ) via

$$
R_{\mathrm{W}}^{\mathrm{c}}=\frac{1}{\left(1+F_{0}^{s}\right)}= \begin{cases}1 ; & \text { for free fermions } \\ 4 ; & \text { for pairs } \\ 9 ; & \text { for trions }\end{cases}
$$

From the equation (E5) and (E6) we could calculate the relation between Fermi speed and sound velocity. For example, the velocities of trions, pairs, and single fermionic atoms are given by

$$
v^{3}=\frac{1}{3} v_{F}^{(3)} ; v^{2}=\frac{1}{2} v_{F}^{(2)} ; v^{1}=v_{F}^{(1)},
$$

respectively. This builds up an intrinsic connection between the Luttinger liquid and Fermi liquid.

Furthermore, from the free fermion nature of the Fermi liquid, we can express the specific heat in terms of these sound velocities

$$
\frac{c_{V}}{T}=\frac{\pi}{3}\left(\frac{1}{v_{F}^{1}}+\frac{2}{v_{F}^{2}}+\frac{3}{v_{F}^{3}}\right)=\frac{\pi}{3}\left(\frac{1}{v^{1}}+\frac{1}{v^{2}}+\frac{1}{v^{3}}\right),
$$

where we have dropped the unit $k_{B}^{2} \hbar^{-1}$; here the interaction effect is encoded in the velocities $v^{1,2,3}$. This equation could be obtained by considering the leading term of the TBA equations at low temperature region. This shows a consistency of our derivation by the 1D Fermi-liquid-like description. The derivation above could be extended to the $\mathrm{SU}(\mathrm{w})$ attractive Fermi gases in a straightforward way. All the derivation above could be directly extended to the $\mathrm{SU}(\mathrm{w})$ attractive Fermi gases:

$$
\frac{c_{V}}{T}=\frac{\pi}{3}\left(\frac{1}{v^{1}}+\frac{1}{v^{2}}+\cdots+\frac{1}{v^{w}}\right) .
$$

We can also prove this additivity rule using the TBA equations, see Appendix B.
[1] S. Sachdev, Quantum Phase transitions (Cambridge University Press, Cambridge, 1999).

[2] C. J. Pethick and H. Smith, Bose-Einstein Condensation in Dilute Gases (Cambridge University Press, Cambridge, England, 2008).

[3] A. C. Hewson, The Kondo Problem to Heavy Fermions (Cambridge University Press, Cambridge, 1997).

[4] T. Giamarchi, Quantum Physics in one dimension (Oxford University Press, Oxford, 2004).

[5] F. H. L. Essler, H. Frahm, F. Göhmann, A. Klümper, and V. E. Korepin, The One-Dimensional Hubbard Model (Cambridge University Press, Cambridge, 2005).

[6] M. A. Cazalilla, R. Citro, T. Giamarchi, E. Orignac, and M. Rigol, One dimensional bosons: From condensed matter systems to ultracold gases, Rev. Mod. Phys. 83, 1405 (2011).
[7] A. Imambekov, T. L. Schmidt, and L. I. Glazman, One-dimensional quantum liquids: Beyond the Luttinger liquid paradigm, Rev. Mod. Phys. 84, 1253 (2012).

[8] G. Wiedemann and R. Franz, Ueber die WärmeLeitungsfähigkeit der Metalle, Ann. Phys. 89, 497 (1853).

[9] K. Kadowaki and S. B. Woods, Universal relationship of the resistivity and specific heat in heavyFermion compounds, Solid State Commun. 58, 507 (1986).

[10] A. C. Jacko, J. O. Fjaerestad, and B. J. Powell, A unified explanation of the Kadowaki-Woods ratio in strongly correlated metals, Nat. Phys. 5, 422 (2009).

[11] M. A. Tanatar, J. Paglione, C. Petrovic, and L. Taillefer, Anisotropic violation of the Wiedemann-Franz law at a quantum critical point, Science 316, 1320 (2007). 
[12] M. Taupin, G. Knebel, T. D. Matsuda, G. Lapertot, Y. Machida, K. Izawa, J.-P. Brison, and J. Flouquet, Thermal Conductivity Through The Quantum Critical Point in $\mathrm{YbRh}_{2} \mathrm{Si}_{2}$ at very low Temperature, Phys. Rev. Lett. 115, 046402 (2015).

[13] A. Sommerfeld, Zur Elektronentheorie der Metalle auf Grund der Fermischen Statistik (Electron theory on the basis of the Fermi statistics), Z. Phys. 47, 1 (1928).

[14] K. G. Wilson, The renormalization group: Critical phenomena and the Kondo problem, Rev. Mod. Phys. 47, 773 (1975).

[15] A. J. Schofield, Non-Fermi liquids, Contemp. Phys. 40, 95 (1999).

[16] Y.-P. Wang, Fermi liquid features of the one-dimensional Luttinger liquid, Int. J. Mod. Phys. B 12, 3465 (1998).

[17] K. Ninios, T. Hong, T. Manabe, C. Hotta, S. N. Herringer, M. M. Turnbull, C. P. Landee, Y. Takano, and H. B. Chan, Wilson Ratio of a Tomonaga-Luttinger Liquid in a Spin-1/2 Heisenberg Ladder, Phys. Rev. Lett. 108, 097201 (2012).

[18] X.-W. Guan, X.-G. Yin, A. Foerster, M. T. Batchelor, C.-H. Lee, and H. Q. Lin, Wilson ratio of Fermi gases in one dimension, Phys. Rev. Lett. 111, 130401 (2013).

[19] B. Yang, Y.-Y. Chen, Y.-G. Zheng, H. Sun, H.-N. Dai, X.-W. Guan, Y.-Z. Yuan, and J.-W. Pan, Observation of quantum criticality and Luttinger liquid in one-dimensional Bose gases, arXiv:1611.00426.

[20] T. L. Ho and S. Yip, Pairing of Fermions with Arbitrary Spin, Phys. Rev. Lett. 82, 247 (1999).

[21] C. Wu, J.-P. Hu, and S.-C. Zhang, Exact SO(5) Symmetry in the Spin-3/2 Fermionic System, Phys. Rev. Lett. 91, 186402 (2003).

[22] M. A. Cazalilla and A. M. Rey, Ultracold fermi gases with emergent SU(N) symmetry, Rep. Prog. Phys. 77, 124401 (2014).

[23] I. Boettcher, L. Bayha, D. Kedar, P. A. Murthy, M. Neidig, M. G. Ries, A. N. Wenz, G. Zürn, S. Jochim, and T. Enss, Equation of State of Ultracold Fermions in the 2d BEC-BCS Crossover, Phys. Rev. Lett. 116, 045303 (2016).

[24] S. Taie, R. Yamazaki, S. Sugawa, and Y. Takahashi, An SU(6) Mott insulator of an atomic fermi gas realized by large-spin Pomeranchuk cooling, Nat. Phys. 8, 825 (2012).

[25] F. Scazza, C. Hofrichter, M. Höfer, P. C. De Groot, I. Bloch, and S. Fölling, Observation of two-orbital spin-exchange interactions with ultracold SU(N)-symmetric fermions, Nat. Phys. 10, 779 (2014).

[26] Y. Kono, T. Sakakibara, C. P. Aoyama, C. Hotta, M. M. Turnbull, C. P. Landee, and Y. Takano, Field-Induced Quantum Criticality and Universal Temperature Dependence of the Magnetization of a Spin-1/2 Heisenberg Chain, Phys. Rev. Lett. 114, 037202 (2015).

[27] X.-W. Guan, M. T. Batchelor, and C.-H. Lee, Fermi gases in one dimension: From Bethe ansatz to experiments, Rev. Mod. Phys. 85, 1633 (2013).

[28] M. Takahashi, Thermodynamics of One-Dimensional Solvable Models (Cambridge University Press, Cambridge, 1999).

[29] P. Schlottmann, Thermodynamics of the one-dimensional multicomponent fermi gas with a delta-fulction interaction, J. Phys.: Condens. Matter 5, 5869 (1993).

[30] X.-W. Guan, M. T. Batchelor, C. Lee, and M. Bortz, Phase transitions and pairing signature in strongly attractive Fermi atomic gases, Phys. Rev. B 76, 085120 (2007).

[31] B. Sutherland, Further Results for the Many-Body Problem in one Dimension, Phys. Rev. Lett. 20, 98 (1968).
[32] M. Takahashi, Ground state energy of the one-dimensional electron system with short-range interaction. I, Prog. Theor. Phys. 44, 348 (1970); Many-body problem of attractive fermions with arbitrary spin in one dimension, ibid. 44, 899 (1970).

[33] M. Olshanii, Atomic Scattering in the Presence of an External Confinement and a Gas of Impenetrable Bosons, Phys. Rev. Lett. 81, 938 (1998).

[34] G. Pagano, M. Mancini, G. Cappellini, P. Lombardi, F. Schäfer, H. Hu, X.-J. Liu, J. Catani, C. Sias, M. Inguscio, and L. Fallani, A one-dimensional liquid of fermions with tunable spin, Nat. Phys. 10, 198 (2014).

[35] Y.-A. Liao, A. S. C. Rittner, T. Paprotta, W.-J. Li, G. B. Partridge, R. G. Hulet, S. K. Baur, and E. J. Mueller, Spin-imbalance in a one-dimensional Fermi gas, Nature (London) 467, 567 (2010).

[36] A. N. Wenz, G. Zürn, S. Murmann, I. Brouzos, T. Lompe, and S. Jochim, From few to many: Observing the formation of a Fermi sea one atom at a time, Science 342, 457 (2013).

[37] C.-N. Yang, Some Exact Results for the Many-Body Problem in one Dimension with Repulsive Delta-Function Interaction, Phys. Rev. Lett. 19, 1312 (1967).

[38] M. Gaudin, Un systeme a une dimension de fermions en interaction, Phys. Lett. A 24, 55 (1967).

[39] G. Orso, Attractive Fermi Gases with Unequal Spin Populations in Highly Elongated Traps, Phys. Rev. Lett. 98, 070402 (2007).

[40] H. Hu, X.-J. Liu, and P. D. Drummond, Phase Diagram of a Strongly Interacting Polarized Fermi Gas in one Dimension, Phys. Rev. Lett. 98, 070403 (2007).

[41] S. Capponi, G. Roux, P. Lecheminant, P. Azaria, E. Boulat, and S. R. White, Molecular superfluid phase in systems of onedimensional multicomponent fermionic cold atoms, Phys. Rev. A 77, 013624 (2008).

[42] P. Gegenwart, Q. Si, and F, Steglich, Quantum criticality in heavy-fermion metals, Nat. Phys. 4, 186 (2008).

[43] X.-W. Guan, M. T. Batchelor, C. Lee, and H.-Q. Zhou, Magnetic Phase Transitions in One-Dimensional Strongly Attractive Three-Component Ultracold Fermions, Phys. Rev. Lett. 100, 200401 (2008).

[44] C. C. N. Kuhn and A. Foerster, Phase diagrams of threecomponent attractive ultracold fermions in one dimension, New J. Phys. 14, 013008 (2012).

[45] J.-Y. Lee, X.-W. Guan, and M. T. Batchelor, Yang-Yang method for the thermodynamics of one-dimensional multi-component interacting fermions, J. Phys. A: Math. Theor. 44, 165002 (2011).

[46] I. Affleck and F. D. M. Haldane, Phys. Rev. B 36, 5291 (1987).

[47] H. Frahm and V. E. Korepin, Phys. Rev. B 42, 10553 (1990); 43, 5653 (1991).

[48] C. H. Cheng and S. K. Yip, Trapped resonant fermions above the superfluid transition temperature, Phys. Rev. B 75, 014526 (2007).

[49] T. L. Ho and Q. Zhou, Obtaining the phase diagram and thermodynamic quantities of bulk systems from the densities of trapped gases, Nat. Phys. 6, 131 (2010).

[50] M. R. Andrews, D. M. Kurn, H.-J. Miesner, D. S. Durfee, C. G. Townsend, S. Inouye, and W. Ketterle, Propagation of Sound in a Bose-Einstein Condensate, Phys. Rev. Lett. 79, 553 (1997). 